\title{
Estimativa da altura da base das copas com o uso de dados laser scanning aerotransportado (LiDAR)
}

\section{Estimation of canopy base height using airborne laser scanning data (LiDAR)}

\author{
Marcos Giongo $^{1 *}$, Henrique Soares Koehler ${ }^{2}$, Marcelo Ribeiro Viola ${ }^{1}$, Patrick dos Santos \\ Bastos $^{1}$; André Ferreira dos Santos $^{1}$ e Giovanni Santopuoli ${ }^{3}$
}

\begin{abstract}
${ }^{1}$ Engenharia Florestal, Universidade Federal do Tocantins, Gurupi, TO - Brasil. ${ }^{2}$ Engenharia Florestal, Universidade Federal do Paraná, Curitiba, PR - Brasil. ${ }^{3}$ Scienze Forestali e Ambientali, Università degli Studi del Molise, Pesche-IS - Itália.
\end{abstract}

\begin{abstract}
Lately, data acquisition using Airborne Laser Scanning (ALS) with LiDAR technology (Light Detection and Ranging) is becoming promising in the forest field, especially for estimation of dendrometric variables and to evaluate vertical and horizontal structure of the forest. Topographic and forest coverage information are extremely important to forest and natural resources managers. Accurate information on trees height and density are fundamental for planning, but also hard to obtain by conventional methods. The use of modeling associated with LIDAR data allows the researcher to obtain estimates of several other forest variables, such as basal area, diameter, volume, biomass and combustible material. The estimation of the trees base heights with plots of different sizes $(10,15$ and 20 meters) showed an standard error of 1.42, 0.95 and $0.82 \mathrm{~m}$, which correspond to 23.62, 15.70 and $13.84 \%$, respectively.
\end{abstract}

Key-words: LiDAR, precision forest inventory, forest fire

\section{INTRODUÇÃO}

A composição florestal e sua estrutura representam informações importantes e necessárias para nortear o manejo florestal de uso múltiplo, entretanto são difíceis de serem obtidas em grandes áreas florestais.

Nos estudos relacionados aos incêndios florestais existe uma série de variáveis importantes e necessárias para serem inseridas nos modelos de comportamento do fogo, como por exemplo, o FARSITE (Finney, 1998) e BehavePlus (Andrews, 2009), onde é possível estimar as áreas em que um fogo de superfície provavelmente possa transforma-se em incêndio de copa. Assim, entre as diversas variáveis, a altura da base das copas da cobertura florestal é uma das variáveis de grande importância nestes modelos.

Em inglês, a altura da base das copas da cobertura florestal, é denominada como Canopy Base Height $(\mathrm{CBH})$. A sua definição em literatura não é muito ciara, apresentandio aỉgumas variações, o que muitas vezes torna difícil sua utilização (Mitsopoulos e Dimitrakopoulos, 2007). McAlpine e Hobbs (1994) definem a CBH como a distância entre o piso da floresta à base da copa viva (Figura 1). Segundo Van Wagner (1993); Scott e Reinhardt (2001) a CBH pode ser definida como a menor altura em que há uma quantidade suficiente de combustível para a propagação do fogo verticalmente, para a copa das árvores.

Tradicionalmente as estimativas de variáveis relacionadas à altura das bases das copas das árvores em estudos de comportamento do fogo têm sido obtidas em levantamentos de campo utilizando técnicas de amostragem. Entretanto, as técnicas baseadas em medições de campo muitas vezes não são padronizadas e a aplicação dos valores estimados fora do limite das áreas amostradas pode gerar resultados pouco satisfatórios e desta forma comprometendo a avaliação do comportamento do fogo, principalmente em áreas.

Author for correspondence: giongo@uft.edu.br 


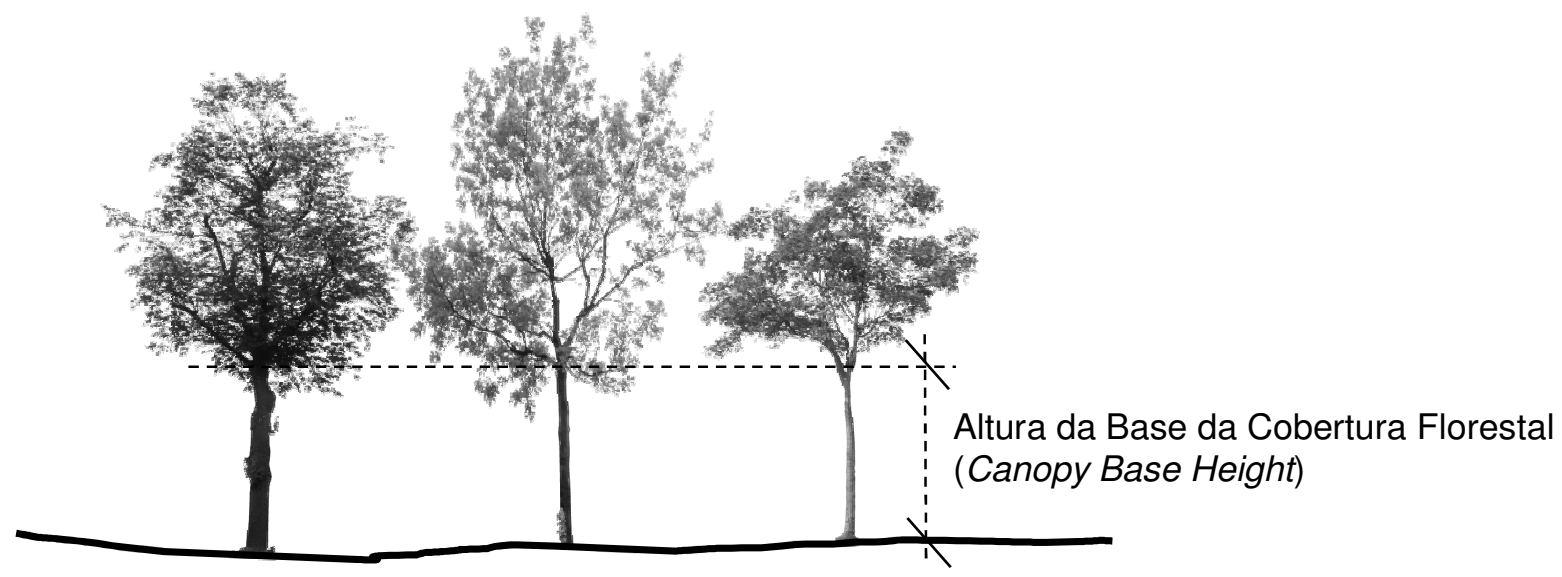

Figura 1 - Altura da Base da Cobertura Florestal (Canopy Base Height).

Atualmente o uso de técnicas e ferramentas baseadas em sensoriamento remoto, como o Sistema de Posicionamento Global (GPS) e Sistemas de Informação Geográfica (SIG) vem demonstrando grande auxílio na coleta e na manipulação de informações de variáveis florestais. Segundo Roff et al. (2005) existe necessidade de uma abordagem padronizada e eficiente para medir as variáveis relacionadas aos combustíveis aéreos em áreas florestais, que apresentam uma grande variedade de características estruturais. O uso de técnicas de sensoriamento remoto normalmente necessita ser integrado com as informações obtidas em levantamento de campo e sequencialmente é também necessário o uso de uma abordagem estatística robusta para maximizar a precisão da modelagem do comportamento do fogo.

Mais recentemente com o desenvolvimento e a disponibilidade de dados de sensores ativos, como por exemplo, o RADAR e o LiDAR, surgiu à possibilidade de estimativa direta de variáveis florestais, mesmo no sub-bosque florestal, como a altura da base da cobertura florestal, onde é possível realizar estimativas diretamente dos dados fornecidos por estes sensores, sem a necessidade de levantamentos em campo.

Andersen et al. (2004), em uma área florestal de coníferas, no oeste do estado de Washington nos Estados Unidos, utilizaram dados de RADAR de abertura sintética interferométrico (Interferometric Synthetic Aperture Radar - IfSAR) obtidos com o uso do sistema TopoSAR para estimar variáveis florestais de interesse para a modelagem do comportamento do fogo. A determinação da altura da base das copas das árvores foi baseada em conceitos definidos nos trabalhos de Scott e Reinhardt (2001), onde a altura da base da copa é calculada como a menor altura do dossel florestal no quale a densidade de combustível é superior a um limite crítico de $0,011 \mathrm{~kg} / \mathrm{m}^{3}$. Utilizando uma análise de regressão múltipla foi demonstrado que as estimativas dos dados obtidos do IfSAR apresentaram alta correlação com medidas dos atributos florestais em campo, com destaque para a altura do dossel $\left(\mathrm{R}^{2}=0,89\right)$, altura da base da copa $\left(R^{2}=0,85\right)$, densidade da copa $\left(R^{2}=0,74\right)$ e o peso do combustível do dossel $\left(\mathrm{R}^{2}=0,77\right)$.

$\mathrm{O}$ avanço da tecnologia laser scanning nos últimos anos permitiu um aumento de sua capacidade de coleta de dados e a possibilidade de diferenciação e registro dos diversos retornos do impulso laser, possibilitando o uso das informações obtidas para a estimativa de uma grande variedade de atributos florestais (Zimble et al., 2003). Dentre os diversos atributos estimados diretamente dos dados da varredura laser, tem-se a altura da base da cobertura florestal, que é variável de grande importância em estudos relacionados à estrutura de áreas florestais e principalmente em estudos relacionados ao comportamento e planejamento de combate a incêndios florestais.

Næsset e Økland (2002) avaliaram o potencial do uso de dados da varredura laser scanning aerotranportada, em áreas de floresta boreal, localizadas ao sudeste da Noruega, para a estimativa de variáveis relacionadas à altura e dimensões de copa de indivíduos arbóreos. Os resultados demonstraram coeficiente de determinação $\left(R^{2}\right)$ entre as estimativas dos dados LiDAR com os resultados obtidos em 
levantamentos de campo, que variaram de 0,47 a 0,91 dentre as diversas variáveis estudas.

Morsdorf et al. (2004) avaliaram a possibilidade de estimativa de variáveis de árvores isoladas a partir dos dados LiDAR na parte oriental do vale Ofenpass, dentro de um Parque Nacional na Suíça, com a utilização de uma segmentação baseada no $k$-means clustering. A regressão linear da medição da altura das árvores resultou em um $\mathrm{R}^{2}$ ajustado de 0,92 em relação aos valores obtidos em levantamento de campo. Com base nas variáveis estimadas foi realizada uma reconstrução geométrica da floresta por um modelo parabolóide, utilizando os valores de posição das árvores, a altura, diâmetro da copa e altura da base das copas. Riaño et al. (2003) aplicaram dados da varredura laser aerotransportada para estimar os parâmetros florestais necessários para a modelagem do comportamento do fogo em uma área florestal localizada a $2 \mathrm{~km}$ a leste de Ravensburg, no sudoeste da Alemanha. Dentre as variáveis estimadas, foi avaliada a altura da base das copas das árvores, através de uma metodologia baseada no percentual da altura dos retornos dos pulsos laser. Andersen et al. (2005) em uma área $5,2 \mathrm{~km}^{2}$, no estado de Washington, Estados Unidos, realizaram estudo para estimativa de parâmetros do combustível do dossel florestal com base em dados LIDAR com uma densidade de 3,52 pulsos.m $\mathrm{m}^{-2}$. Neste trabalho foram encontradas fortes correlações entre as estimativas dos dados da cobertura florestal obtidas dos dados da varredura laser com as informações coletadas nos levantamentos de campo, tais como: peso da coroa de combustível $\left(R^{2}=0,86\right)$, densidade de copa $\left(R^{2}\right.$ $=0,84)$, altura da base da copa $\left(\mathrm{R}^{2}=0,77\right)$ e altura do dossel $\left(\mathrm{R}^{2}=0,98\right)$.

Neste sentido, o trabalho desenvolvido teve como objetivo principal avaliar a possibilidade da estimativa da altura da base das copas das árvores com o uso de dados obtidos da varredura laser aerotransportada (LiDAR) em uma área florestal com alta densidade de indivíduos arbóreos.

\section{MATERIAL E MÉTODOS Localização da área de estudo}

A área de estudo localiza-se na Região Sul do Brasil, na porção leste do estado do Paraná, na cidade de Curitiba, entre as coordenadas $25^{\circ} 27^{\prime} 06^{\prime \prime} \mathrm{S} / 4^{\circ} 14^{\prime} 37^{\prime \prime} \mathrm{W}$ e $25^{\circ} 26^{\prime} 40^{\prime \prime} \mathrm{S}$ / $49^{\circ} 14^{\prime} 10^{\prime \prime} \mathrm{W}$ (Figura 2). A altitude é de aproximadamente 900 m s.n.m., apresentando um clima subtropical úmido mesotérmico $(C f b)$ sem estação seca definida, com temperatura média no mês mais quente de $22^{\circ} \mathrm{C}$ e de $12^{\circ} \mathrm{C}$ no mês mais frio (Peel et al., 2007). A média anual para o índice pluviométrico é de $1.450 \mathrm{~mm}$ e para a umidade relativa é de $81 \%$.
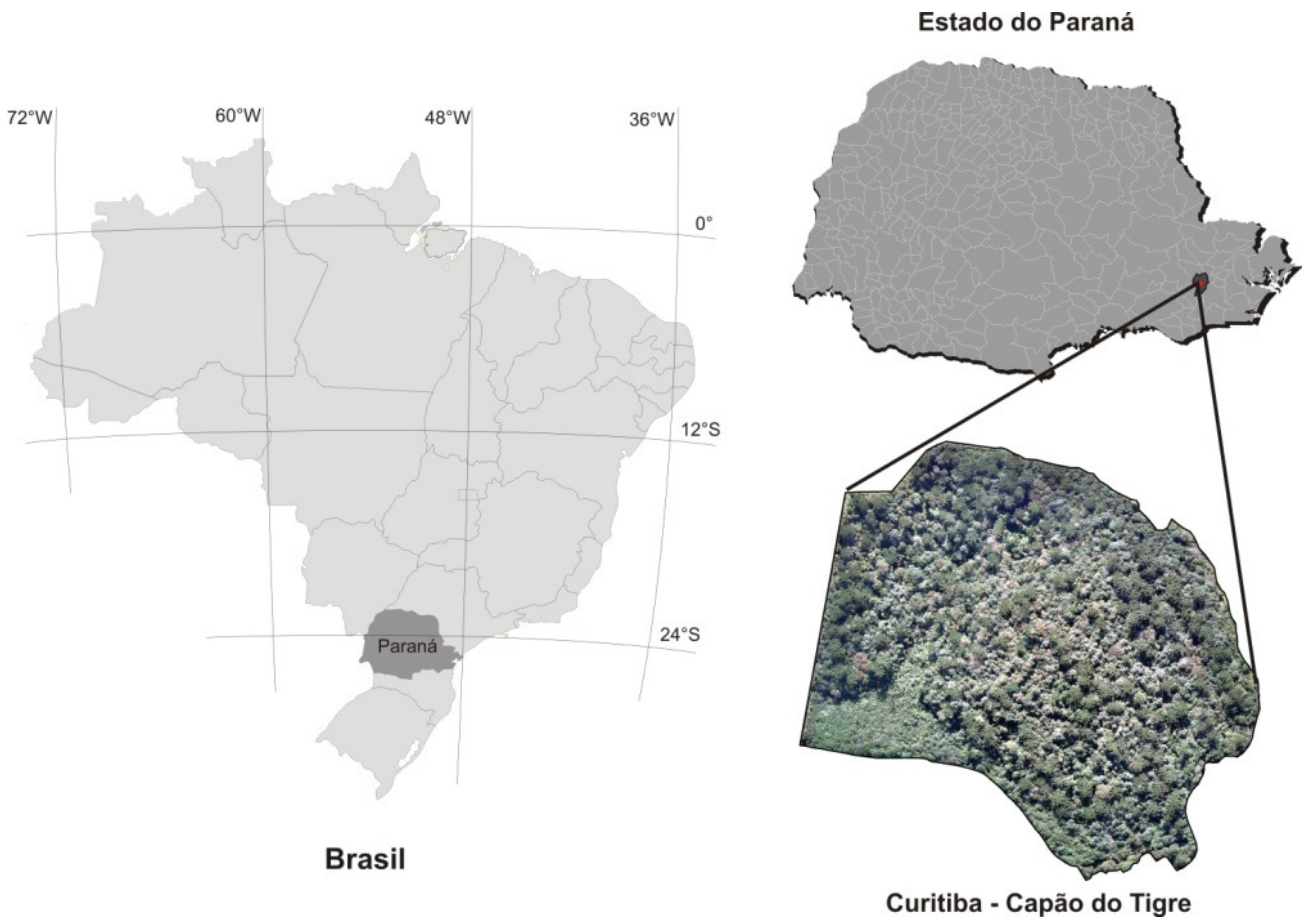

Figura 2 - Localização da área de estudo. 
Levantamentos de campo e características da cobertura florestal

Inicialmente a área de estudo foi dividida em "blocos" com o uso de uma grade de 50 metros em toda sua extensão, utilizando como referência o sistema de coordenadas UTM (Datum SAD 69). Cada bloco obtido foi codificado utilizando uma letra e um número, como se pode observar na Figura 3. O uso do sistema de coordenada UTM teve como objetivo facilitar os trabalhos de campo e permitir que as informações resultantes do inventário florestal fossem obtidas de forma georreferenciada, permitindo assim a realização de avaliações das estimativas obtidas a partir das informações provenientes da varredura laser.

Ao total, em toda a área de estudo, foram obtidos 80 blocos, sendo um bloco selecionado para a realização de um censo dos indivíduos arbóreos. A escolha do bloco a ser inventariado foi feita de forma aleatória com a restrição de que o mesmo não pudesse pertencer ao grupo dos blocos que se encontravam nos limites da área de estudo. Esta restrição foi utilizada para que o bloco apresentasse em toda a sua extensão cobertura florestal e características estruturais representativas da área de estudo.

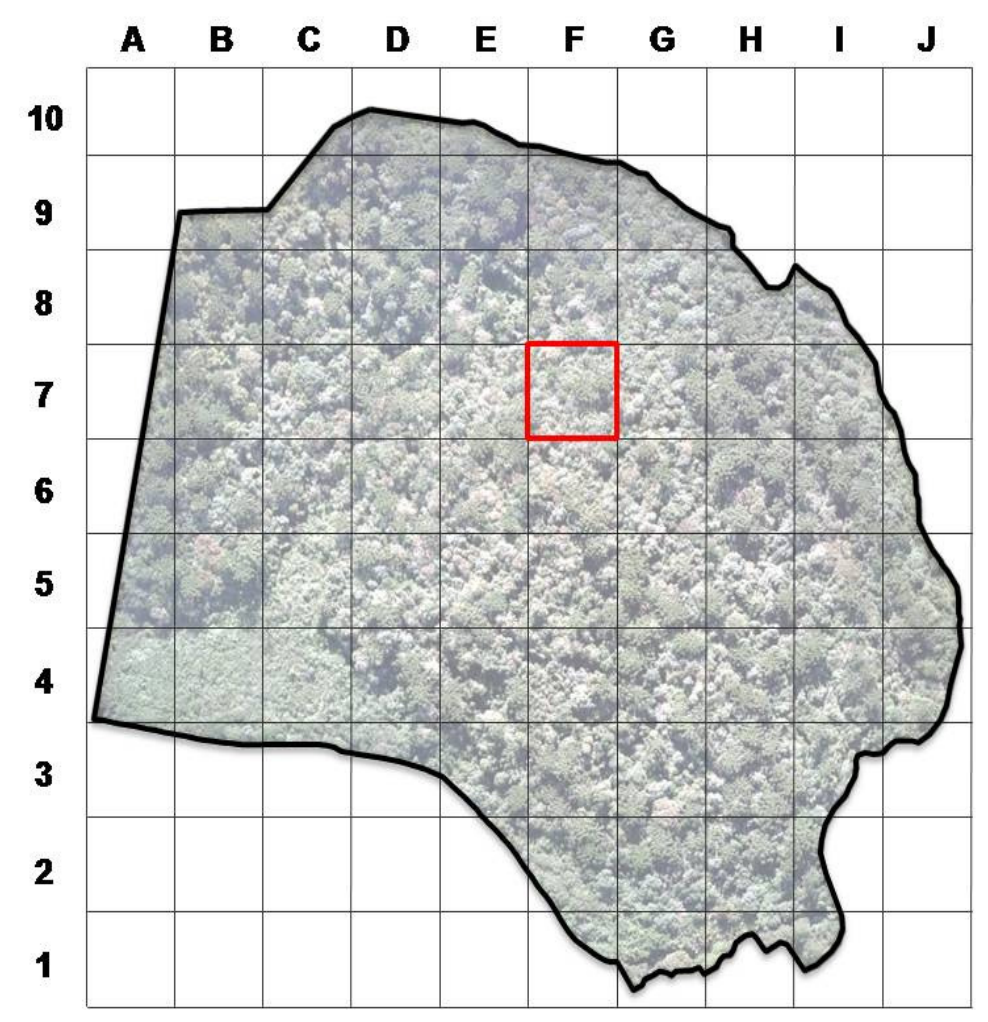

Figura 3 - Divisão e localização dos blocos na área de estudo.

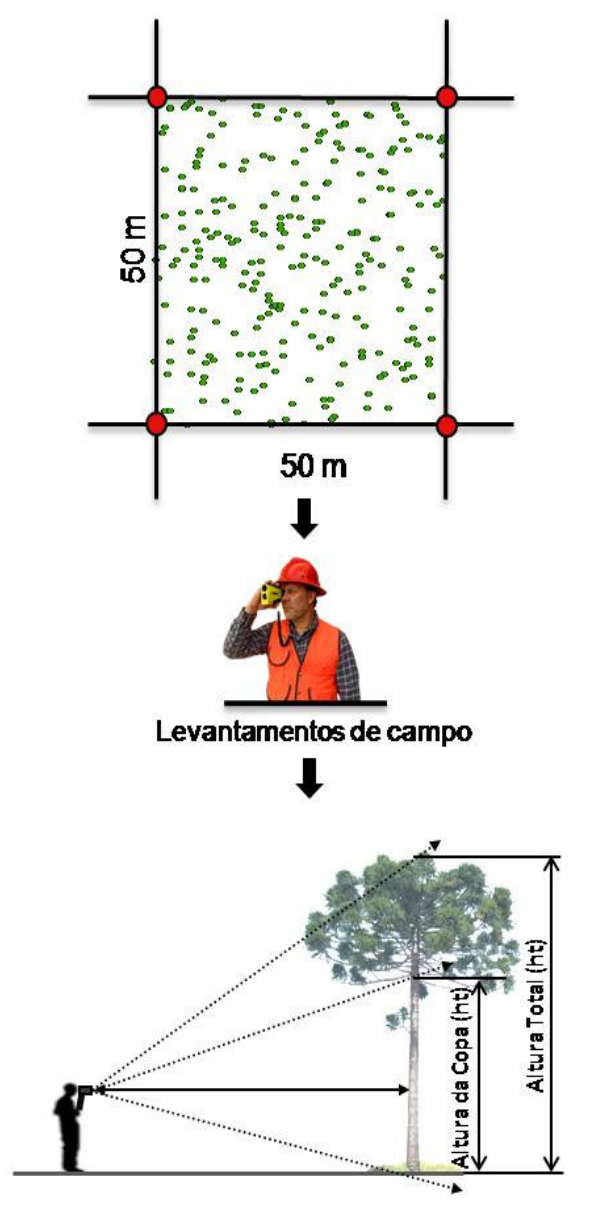

Durante o inventário florestal realizado, as árvores foram georrefenciadas utilizando como referência os piquetes previamente instalados e georeferenciados nas extremidades de cada bloco. Além das informações espaciais das árvores individuais, foram também coletadas diversas informações dos indivíduos arbóreos, tais como: diâmetro (DAP), altura total $\left(h_{t}\right)$, altura da base da copa $\left(h_{b c}\right)$, raio da copa $\left(C_{r}\right)$, espécie, estrato arbóreo, estado fitossanitário, qualidade do fuste e tipo de copa. Na Tabela 1 são apresentados os principais resultados obtidos com o inventário florestal. 
Tabela 1 - Principais variáveis dendrométricas obtidas no inventário florestal.

\begin{tabular}{lccccc}
\hline \multirow{2}{*}{ Variável dendrométrica } & \multirow{2}{*}{ Unidade } & \multicolumn{3}{c}{ Valores } & Desvio \\
\cline { 3 - 5 } & & Médio & Máximo & Mínimo & padrão \\
\hline Diâmetro (DAP) & $c m$ & 15,74 & 68,50 & 10,03 & 8,45 \\
Área basal $(G)$ & $m^{2} \cdot h a^{-1}$ & 27,32 & -- & -- & -- \\
Altura total $\left(h_{t}\right)$ & $m$ & 10,78 & 24,50 & 4,40 & 2,86 \\
Altura da base da copa $\left(h_{b c}\right)$ & $m$ & 6,08 & 19,30 & 1,50 & 2,64 \\
Tamanho da copa $\left(C_{h}\right)$ & $m$ & 4,68 & 12,70 & 1,00 & 2,42 \\
Raio da copa $\left(C_{r}\right)$ & $m$ & 1,95 & 8,45 & 0,61 & 0,93 \\
Área da copa $\left(C_{a}\right)$ & $m^{2}$ & 14,69 & 224,45 & 1,16 & 19,89 \\
\hline
\end{tabular}

$\mathrm{Na}$ parcela inventariada foram identificadas 47 espécies arbóreas, em que as espécies com maior frequência foram: Cafezeiro (Casearia sylvestris SW.), Symplocos tetrandra (Mart.) Miq., Vacum (Allophylus edulis (A. St-Hil., Cambess. \& A. Juss.)) e Açoita cavalo (Luehea divaricata Mart.) com respectivamente com 16, 12, 8 e $7 \%$.

\section{Dados LiDAR}

Os dados da varredura laser na área de estudo foram adquiridos através do uso de um sistema LiDAR aerotransportado fabricado pela empresa canadense Optech Inc., modelo ALTM 2050 (Airborne Laser Terrain Mapper). Este modelo apresenta uma frequência de perfilamento de até $50 \mathrm{kHz}$ (ou seja, um registro de até $50 \mathrm{mil}$ pontos/segundo), pode ser operado em altitudes de 210 até 2.000 metros com um ângulo de varredura podendo variar de 0 a $\pm 20^{\circ}$ onde registra o primeiro (first) e o último (last) pulso de retorno. O levantamento LiDAR na área de estudo foi realizado com uma altitude de vôo de aproximadamente 1.000 metros, com uma velocidade média de $150 \mathrm{~km} / \mathrm{h}$, com um ângulo de varredura de $15^{\circ}$ e um diâmetro do feixe laser a terra (footprint) de $0,27 \mathrm{~m}$.

\section{Elaboração dos dados LiDAR e do inventário florestal}

Inicialmente foi elaborado um arquivo vetorial dos limites da área de estudo o qual foi utilizado para extrair os dados da varredura laser scanning de interesse. A extração da "nuvem de pontos" georeferenciada dentro da área de estudo teve como objetivo principal reduzir a quantidade de dados LiDAR e otimizar os procedimentos para extração das variáveis de interesse.

Com a nuvem de pontos LiDAR recortada, o primeiro procedimento foi à obtenção da altitude em relação ao terreno de cada um dos pontos da varredura laser. Para este procedimento, foram utilizados como referência para a extração das alturas, Modelos Digitais do Terreno (MDT) obtidos também com dados LiDAR. O MDT utilizado no processamento dos dados foi obtido dos dados LiDAR com a utilização de um sistema computacional comercial denominado TerraScan.

Para a estimativa da altura da base das copas das árvores a partir dos dados LiDAR foram utilizadas duas diferentes metodologias com algumas variações ao interno de cada uma das metodologias. A primeira metodologia (1) foi baseada na estimativa da altura da base da copa de árvores individuais $\left(h_{b c}\right)$ e a segunda metodologia (2) baseou-se na estimativa em nível de área, ou seja, altura média da base das copas das árvores $\left(C_{h}\right)$.

Na metodologia 1, a estimativa da $h_{b c}$ foi baseada na identificação dos pontos de menor altura em áreas circulares, onde o centro destas áreas são as posições espaciais dos indivíduos arbóreos, objeto de interesse para a estimativa da $h_{b c}$. Com base nos dados obtidos no inventário florestal (Tabela 1), a altura mínima da $h_{b c}$ foi de $1,50 \mathrm{~m}$, desta forma utilizou-se como restrição que os pontos da varredura laser utilizados na estimativa, não fossem inferiores a $1,40 \mathrm{~m}$. Assim, todos os pontos com altura igual ou inferior a $1,40 \mathrm{~m}$ foram eliminados antes do processo de estimativa da $h_{b c}$. Essa restrição foi inserida para reduzir uma possível influência da vegetação de sub-bosque na estimativa da $h_{b c}$.

A dimensão das áreas circulares utilizadas para as estimativas da $h_{b c}$ foi baseada em dois critérios: o primeiro em área fixa para todas as árvores, utilizando um raio de $1,95 \mathrm{~m}$, o qual corresponde ao raio médio da copa das árvores (inventário florestal); o segundo método utilizou raio variável, com base nas dimensões individuais, ou seja, no raio de copa de cada um dos indivíduos, os quais foram obtidos no levantamento de campo. 
Na metodologia 2, a estimativa da $C_{h}$ por unidade de área, partiu da elaboração de grades de diversas dimensões sobre a área da parcela inventariada. As grades utilizadas apresentaram forma quadrada com dimensões de 10,15 e $20 \mathrm{~m}$, que representaram um total de $100,25,16$ e 9 subparcelas, respectivamente, dentro da parcela inventariada.

Para cada uma das sub-parcelas, foram inicialmente identificados os pontos da varredura laser que pertenciam a mesma e seqüencialmente foi extraído o conjunto de informações das alturas dos pontos em relação ao terreno, obtido em etapa anterior. Com o conjunto de pontos obtidos em cada sub-parcela, nas diferentes dimensões avaliadas, inicialmente foram elaborados histogramas da distribuição dos pontos em função da altura em relação ao terreno e seqüencialmente se elaborou a simulação da onda dos pulsos laser em cada uma das sub-parcelas.

A estimativa da altura média da copa das árvores em cada sub-parcela foi obtida através do uso da onda simulada, onde se identificou a primeira inflexão da curva com a mesma restrição utilizada na metodologia 01 , em que o menor valor não pudesse ser inferior a 1,40 metros. Na Figura 4, é apresentado o fluxograma geral das atividades envolvidas na elaboração dos dados e estimativa das variáveis de interesse.

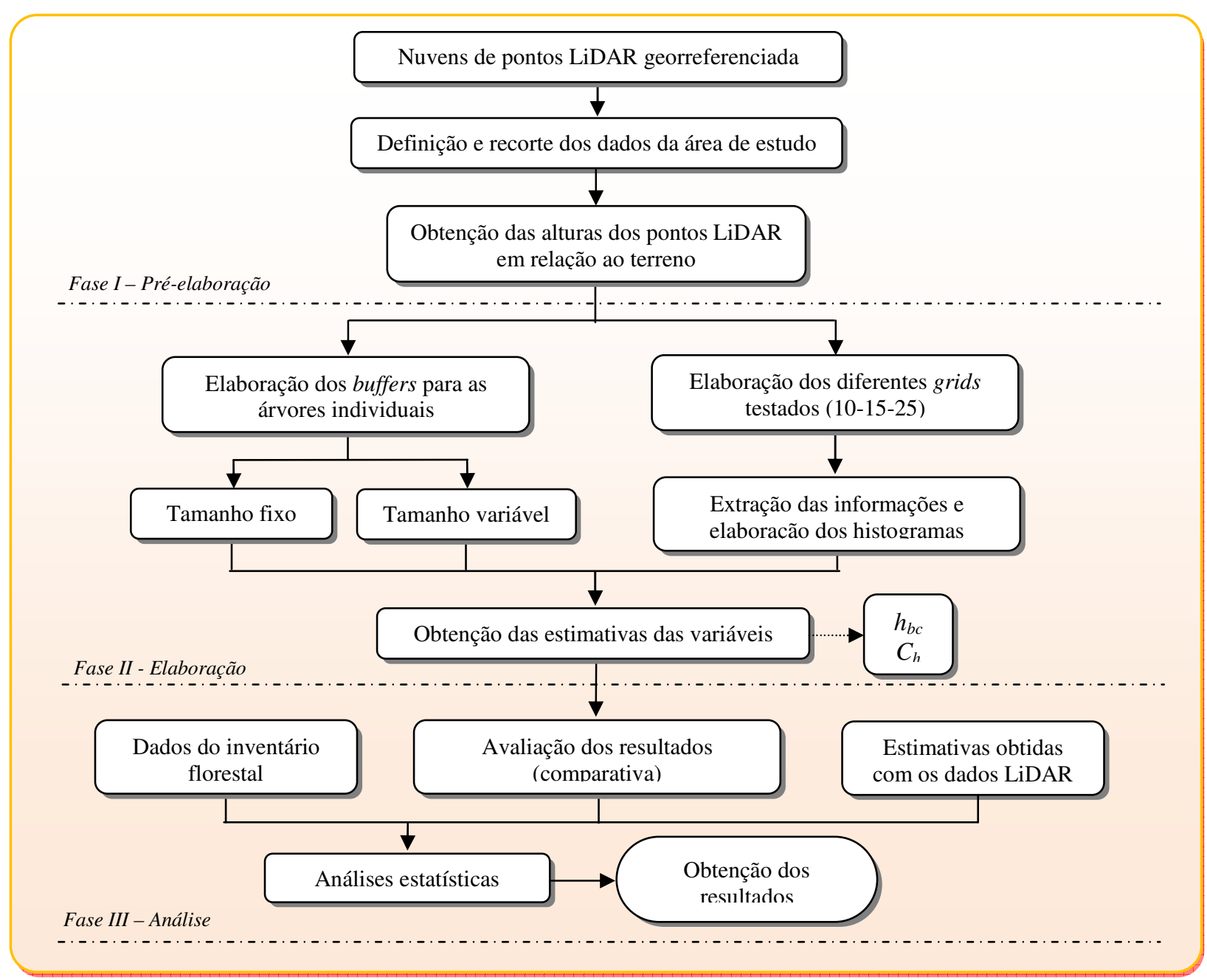

Figura 4 - Fluxograma das metodologias utilizadas para a estimativa da altura da base da copa das árvores.

Nesta metodologia, durante uma fase exploratória dos dados, observou também a possibilidade de estimativa das alturas dos demais extratos arbóreos, medindo as diferentes inflexões da curva. Assim, se procedeu a extração do primeiro ponto de inflexão, do último ponto de inflexão e do ponto intermediário, obtendo desta forma altura de três estratos para cada uma das sub-parcelas avaliada. 
Os dados do inventário florestal foram utilizados para avaliar as estimativas das alturas obtidas dos dados LiDAR. Na metodologia (1), se utilizou diretamente os dados obtidos nos levantamentos de campo, já na metodologia (2), foi necessária uma elaboração dos dados do inventário para que os mesmos pudessem ser utilizados durante o processo de validação das estimativas dos dados LiDAR. Desta forma, para cada uma das subparcelas nas diferentes dimensões de grade avaliadas, foram extraídas as informações da altura média, máxima e mínima das árvores georreferenciadas dentro de cada sub-parcela através de um processo de interpolação dos atributos vetoriais de limite das sub-parcelas.

Com os dados dendrométricos espacializados obtidos durante o levantamento de campo foi possível a elaboração de um modelo digital tridimensional da parcela inventariada, conforme se pode observar na Figura 5. A elaboração tridimensional da floresta teve como objetivo proporcionar uma melhor visualização da estrutura florestal presente, e principalmente uma visualização da distribuição dos dados da varredura laser scanning em função da variação da estrutura florestal presente na área de estudo.
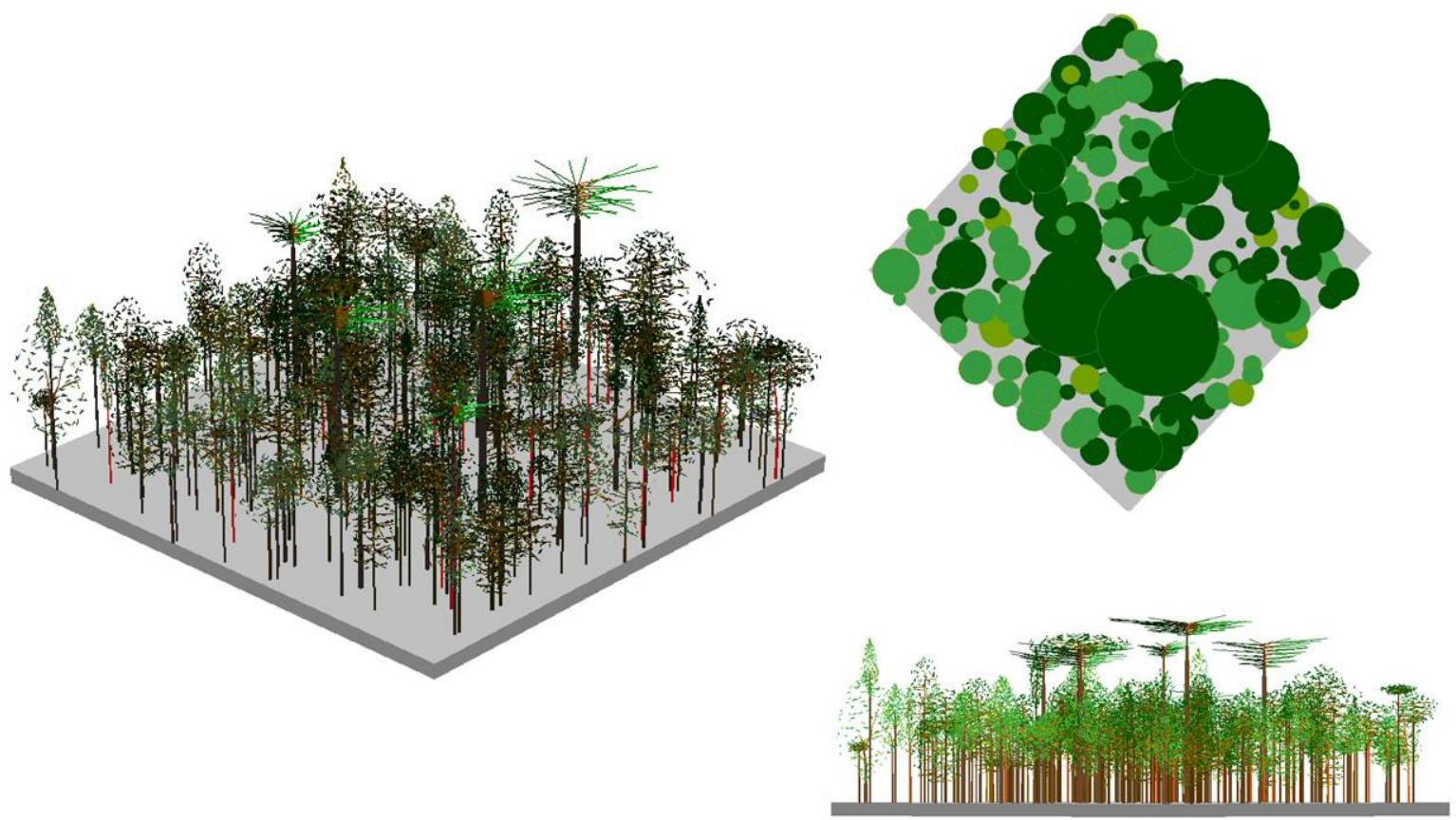

Figura 5 - Estrutura tridimensional do P50 obtido com os dados provenientes do levantamento de campo.

Por fim, nas diferentes metodologias avaliadas foi aplicado o teste Qui-quadrado $\left(\chi^{2}\right)$ para avaliar se a frequência com que a altura da base da copa das árvores estimada a partir dos dados da varredura laser desvia significativamente ou não das estimativas obtidas nos levantamentos de campo com o uso de hipsômetro.

$$
\chi^{2}=\sum \frac{\left(f_{\mathrm{o}}-f_{\mathrm{e}}\right)^{2}}{f_{\mathrm{e}}}
$$

Em que, $f_{\mathrm{o}}$ é a frequência observada e $f_{\mathrm{e}}$ é a frequência esperada.
Seqüencialmente foram calculados os erros médios quadráticos (RMSE) em cada uma das diferentes variações da metodologia utilizada.

$$
\operatorname{RMSE}=\sqrt{\frac{1}{n} \sum_{\mathrm{i}=1}^{n}\left(\hat{\mathrm{Z}}\left(x_{\mathrm{i}}\right)-\mathrm{Z}\left(x_{\mathrm{i}}\right)\right)^{2}}
$$

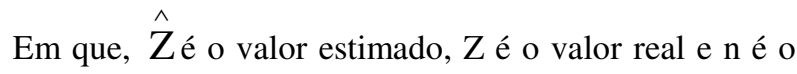
número de observações.

\section{RESULTADOS}

$\mathrm{Na}$ metodologia (1) o teste Qui-quadrado $\left(\chi^{2}\right)$ revelou que as estimativas obtidas dos dados LiDAR apresentam diferenças significativas em relação os valores do inventário florestal. Esta 
situação foi observada para todas as variações utilizadas, ou seja, tanto a metodologia que utilizou a área fixa como a que considerou área variável para a extração da estimativa da altura da base da copa $\left(h_{b c}\right)$ individual.

Quando comparadas as duas variações, a utilização de áreas variáveis apresentou melhores resultados que o uso de área fixa na extração de estimativa das $h_{b c}$ dos dados LiDAR. O método de área fixa apresentou um RMSE de 5,07 m e o uso da área variável um RMSE de 3,60 m. Entretanto, em todas as variações aplicadas na metodologia (1) se observou que a Araucária foi a espécie que apresentou um maior erro na estimativa da $h_{c}$, em todos os casos, apresentando resultado subestimados, com um erro variando de 8,71 a 12,17 metros.

$\mathrm{O}$ uso da metodologia (2) na estimativa da altura média da base das copas das árvores $\left(C_{h}\right)$ a partir dos dados LiDAR demonstrou grande potencialidade da aplicação dos dados deste sensor na estimativa desta variável. Em todas as variações das dimensões do grid $(10,15$ e $20 \mathrm{~m})$ avaliados na metodologia (2) o teste Qui-quadrado $\left(\chi^{2}\right)$ aplicado nas estimativas da altura média da base da copa $\left(C_{h}\right)$ obtidas dos dados LiDAR em relação às médias calculadas para cada célula com o uso de dados obtidos em levantamento de campo com o uso do hipsômetro se aceitou a hipótese da nulidade, ou seja, as frequências observadas não são diferentes das frequências esperadas. Desta forma as estimativas obtidas com a metodologia (2) não apresentaram diferença significativa entre os valores obtidos tradicionalmente para a probabilidade de 0,01 .

O teste Qui-quadrado $\left(\chi^{2}\right)$, na metodologia (2), para as sub-parcelas de 10,15 e 20 m, apresentaram respectivamente os seguintes resultados: $\left(\chi_{\text {cal }}^{2}\right)=9,02 ; 2,41$ e 1,06 com 24,15 e 8 graus de liberdade e um $\left(\chi_{\text {tab }}^{2}\right)=10,85 ; 5,23$ e 1,64 (para uma probabilidade de $99 \%$ ).

Para todas as variações das dimensões das subparcelas utilizadas na metodologia (2) foi também calculado o erro médio quadrático (RMSE), que para as dimensões do grid de 10,15 e $20 \mathrm{~m}$ obteve-se, respectivamente, um RMSE de 1,42; 0,95 e 0,82 m que percentualmente representa um RMSE\% de 23,62; 15,70 e 13,84\%. Desta forma, se pode observar que com o aumento da dimensão das sub-parcelas utilizada para a extração da estimativa da $C_{h}$ houve uma redução significativa do erro da estimativa da variável de interesse
(Figura 6). Isto se deu em função do maior número de pontos laser em cada uma das sub-parcelas, aumentando desta forma a probabilidade que em uma única unidade da sub-parcelas houvesse a presença de pontos em todos os estratos da floresta, ou seja, uma representação mais realista da área.

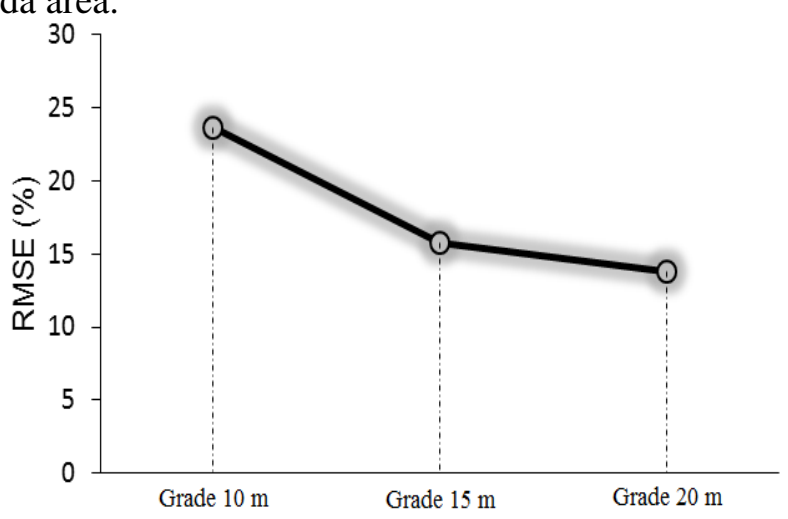

Figura 6 - Evolução do RMSE (\%) nas diferentes variações das dimensões dos grids utilizados na metodologia 2 .

Na metodologia (2) também foi estimado a partir dos dados LiDAR o valor mínimo da altura da base da copa $\left(C_{h m i n}\right)$. Na sub-parcela de $10 \mathrm{~m}$ foi possível obter este valor em $36 \%$ das unidades avaliadas, na sub-parcela de $15 \mathrm{~m}$ em $68,7 \%$, e na sub-parcela de $20 \mathrm{~m}$ foi possível a estimativa desta variável em todas as unidades. Apesar do teste Qui-quadrado $\left(\chi^{2}\right)$ apresentar uma diferença significativa entre as estimativas LiDAR e os dados de campo em todas as variações das dimensões das sub-parcelas na metodologia (2), se observou que na maioria das células foram obtidas estimativas do $C_{\text {hmin }}$ com baixo erro, e a diferença significativa foi dada por algumas unidades isoladas que apresentaram grandes discrepâncias com os valores obtidos em campo. Um exemplo de unidade isolada com valor discrepante pode ser observado na unidade 5 (gride de $20 \mathrm{~m}$ ) apresentada na Figura 7 , onde se obteve uma superestimativa de $2,59 \mathrm{~m}$, com $C_{\text {hmin }}$ de 4,79 e 2,20 m obtidos pelo LiDAR e inventário florestal, respectivamente. 


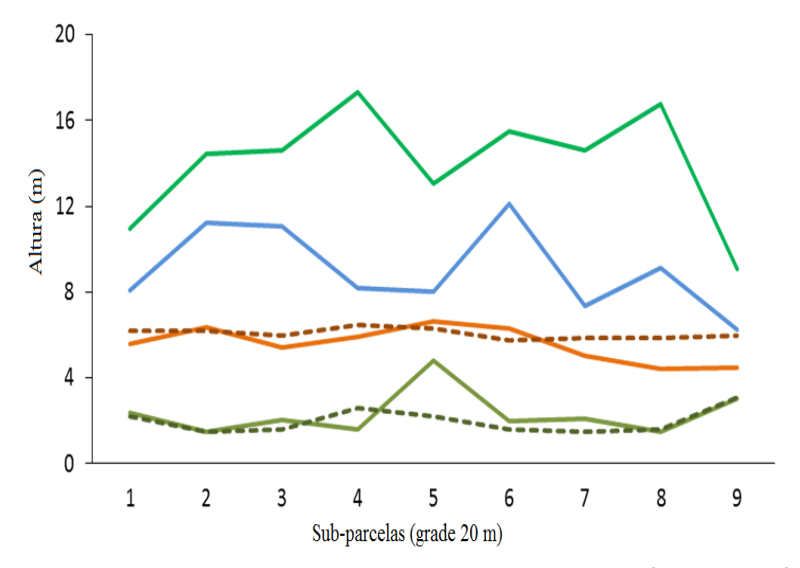

$-\mathrm{C}_{h(\text { min })}-\mathrm{C}_{h(\text { med })}-\mathrm{C}_{h(\text { seg })}-\mathrm{C}_{h(\text { ter })} \ldots \mathrm{C}_{h(\text { min })}{ }_{\text {in }} \ldots \mathrm{C}_{h(\text { med })}$ in

Legenda: $\mathrm{C}_{\mathrm{h}(\min )}$ - Altura mínima da base da copas; $\mathrm{C}_{\mathrm{h}(\mathrm{med})}$ - Altura média da base da copas; $C_{h(s e g)}$ - Altura média base da copas do segundo estrato arbóreo; $\mathrm{C}_{\mathrm{h}(\text { ter })}$ - Altura média base da copas do terceiro estrato arbóreo; $\mathrm{C}_{\mathrm{h}(\min )}$ - Altura mínima base da copas do segundo (inventário florestal) e $C_{h \text { (med) }}$ - Altura média da base da copas (inventário florestal)

Figura 7 - Resultados das estimativas das diversas alturas da base das copas das árvores com a utilização da metodologia (2) com uma grade de $20 \mathrm{~m}$.

\section{CONCLUSÕES}

A metodologia (1), utilizada para a estimativa da altura individual da base da copa $(h c)$ não apresentou resultados satisfatórios. Atribuem-se os maiores erros para as estimativas das árvores de araucária em relação às demais espécies como função principalmente das particulares características da forma de sua copa.

A metodologia aplicada para a estimativa da altura média da base das copas das árvores $\left(C_{\text {hmed }}\right)$ com o uso de dados LiDAR mostrou-se bastante eficiente e de grande potencial de uso, principalmente na aplicação em extensas áreas florestais e de difícil acesso, onde os métodos tradicionais apresentam elevados custos e longo período para a coleta desta informação. Na metodologia (2) observou-se que o aumento da dimensão das células dos grids utilizados para a estimativa proporcionou uma redução do erro quadrático médio (RMSE).

Concluiu-se que a expansão do uso dos dados obtidos de sistemas LiDAR poderá contribuir de forma muito significativa nas atividades de avaliação estrutural de áreas florestais e principalmente auxiliar nos estudos relacionados ao comportamento do fogo e por conseqüência nas atividades de planejamento, combate e prevenção de incêndios florestais.

\section{REFERÊNCIAS}

Andersen, H.-E.; McGaughey, R.; Reutebuch, S.; Schreuder, G.; Agee, J.; Mercer, B. (2004), Estimating canopy fuel parameters in a pacific northwest conifer forest using multifrequency polarimetric IFSAR. Paper presented at the International Archives of Photogrammetry and Remote Sensing, Istanbul, Turkey.

Andersen, H.-E.; McGaughey, R. J.; Reutebuch, S. E. (2005), Estimating forest canopy fuel parameters using LIDAR data Remote Sensing of Environment, 94 (4, 28), 441-449.

Andrews, P. L. (2009), BehavePlus fire modeling system, version 5.0: Variables: Department of Agriculture, Forest Service, Rocky Mountain Research Station.

Finney, M. A. (1998), FARSITE: Fire Area Simulator-model development and evaluation. Ogden, UT: U.S. Department of Agriculture, Forest Service, Rocky Mountain Research Station.

McAlpine, R. S. and Hobbs, M. W. (1994), Predicting the height to live crown base in plantation of four boreal forest species. Journal of International Wildlife, 103-106.

Mitsopoulos, I. D. and Dimitrakopoulos, A. P. (2007), Canopy fuel characteristics and potential crown fire behavior in Aleppo pine (Pinus halepensis Mill.) Annals of Forest Science, 64, 287-299.

Morsdorf, F.; Meiera, E.; Kotza, B.; Ittena, K. I.; Dobbertinc, M.; Allgowerb, B. (2004), LIDARbased geometric reconstruction of boreal type forest stands at single tree level for forest and wildland fire management. Remote Sensing of Environment, 92, 353-362.

Næsset, E., and Økland, T. (2002), Estimating tree height and tree crown properties using airborne scanning laser in a boreal nature reserve. Remote Sensing of Environment, 79, 105-115.

Peel, M. C.; Finlayson, B. L.; McMahon, T. A. (2007), Updated world map of the Köppen-Geiger climate classification. Hydrology and Earth System Sciences - HESSD, 11, 1633-1644.

Riaño, D.; Meier, E.; Allgöwer, B.; Chuvieco, E.; Ustin, S. L. (2003). Modeling airborne laser scanning data for the spatial generation of critical forest parameters in fire behavior modeling. Remote Sensing of Environment, 86, 177-186. 
Roff, A.; Goodwin, N.; Merton, R. (2005), Assessing Fuel Loads using Remote Sensing New South Wales Rural Fire Service Technical Report (pp. 11). Sydney, Autralia: University of New South Wales.

Scott, J. H., and Reinhardt, E. D. (2001), Assessing crown fire potential by linking models of surface and crown fire behavior (Vol. RMRSRP-29, pp. 59). Fort Collins, CO: U.S. Department of Agriculture, Forest Service, Rocky Mountain Research Station.
Van Wagner, C. E. (1993), Prediction of crown fire behavior in two stands of jack pine. Canadian Journal of Research, 23, 442-449.

Zimble, D. A.; Evans, D. L.; Carlson, G. C.; Parker, R. C.; Grado, S. C.; Gerard, P. D. (2003), Characterizing vertical forest structure using small-footprint airborne LiDAR. Remote Sensing of Environment, 87, 171-182. 